\title{
Electron Energy-Loss Spectroscopy of Carbon Films Prepared by Electron-Cyclotron-Resonance Plasma Sputtering
}

\author{
Yoshie Murooka $^{1}$, Nobuo Tanaka ${ }^{2}$, Shigeru Hirono $^{3}$ and Michio Hibino ${ }^{1, *}$ \\ ${ }^{1}$ Center for Integrated Research in Science and Engineering, Nagoya University, Nagoya 464-8603, Japan \\ ${ }^{2}$ Department of Applied Physics, Nagoya University, Nagoya 464-8603, Japan \\ ${ }^{3}$ ECR Plasma Systems Division, NTT AFTY Corporation, Tokyo 181-0013, Japan
}

\begin{abstract}
A protective carbon film, prepared by electron-cyclotron-resonance (ECR) plasma sputtering, has attracted considerable interest because the film can possess both the high wear durability and the high conductivity without doping. Such properties can be obtained, for example, when a negative bias voltage $V_{\mathrm{B}}\left(-40 \leq V_{\mathrm{B}} \leq-140 \mathrm{~V}\right)$ is applied to a substrate made of silicon. Little is known, however, about the atomic structure and bonding state of the ECR films for such a bias region, so that the physical origins of the macroscopic properties are not fully understood. In the present study, electron energy-loss spectroscopy (EELS) in a transmission electron microscope has been applied to investigate such ECR films that were deposited on a sodium chloride substrate for three different bias voltages: $0 \mathrm{~V},-75 \mathrm{~V}$ and $-120 \mathrm{~V}$. The physical density of each ECR film was found to be lower than that of graphitized carbon and that of amorphous carbon. For each of the ECR films, the fraction of sp ${ }^{2}$ bonding was estimated to be more than $90 \%$. The carbon clusters with $\mathrm{sp}^{2}$ bonding were considered to be more ordered for $V_{\mathrm{B}}=-75 \mathrm{~V}$ and $-120 \mathrm{~V}$ than for $V_{\mathrm{B}}=0 \mathrm{~V}$. The averaged density of valence electrons did not change much for each film, but the band structure is considered to vary depending on a local area. For the film prepared by the ECR technique, the macroscopic properties of the film such as electronic and mechanical ones may be controlled by controlling the ordering of $\mathrm{sp}^{2}$ clusters.
\end{abstract}

(Received February 19, 2002; Accepted June 7, 2002)

Keywords: conductive hard carbon film, electron energy-loss spectroscopy, electron-cyclotron-resonance sputtering, sp ${ }^{2}$ fraction, atomic structure

\section{Introduction}

While a variety of techniques have been applied to prepare a protective hard carbon film for hard-disks, the physical origins of the mechanical and electrical properties of the films are still not fully understood. In the present study, such a protective carbon film was prepared by the technique that utilized sputtering in plasma combined with electron-cyclotronresonance (ECR). ${ }^{1)}$ Unlike others, this ECR technique allows flexible tuning of plasma. Using this technique, we recently succeeded in preparing a film that possessed both the wear durability as high as that of bulk diamond and the conductivity as high as that of graphite. ${ }^{2)}$ This was achieved when a negative voltage $V_{\mathrm{B}}\left(-40 \leq V_{\mathrm{B}} \leq 140 \mathrm{~V}\right)$ was applied to the silicon substrate. It was found that the durability depended largely on $V_{\mathrm{B}}$. Despite of the interesting properties, the ECR films obtained in this bias region are not well characterized in atomic dimension.

Electron energy-loss spectroscopy (EELS) combined with transmission electron microscopy (TEM) is a powerful tool to characterize such a thin protective film microscopically; ${ }^{3)}$ especially in a film density, short-range order and atomic bonding. The density of valence electrons $n$ of a thin film can be measured from the low-loss spectrum through the plasmon energy $E_{\mathrm{p}}=\left(4 \pi n / \varepsilon_{0} m\right)^{1 / 2}$, where $\varepsilon_{0}$ is the dielectric constant of the vacuum and $m$ is the effective mass of an electron. ${ }^{3)}$ The fraction of clusters, which have $\mathrm{sp}^{2}$ bonding, can be estimated from the carbon absorption K-edge. ${ }^{4)}$ The degree of $\mathrm{sp}^{2}$ clustering can be reflected also in a low-loss spectrum. These pieces of information are important since they are the keys to control mechanical and electrical properties of

*Present address: Department of Electrical Engineering, Aichi Institute of Technology, Toyota 470-0392, Japan. the protective film.

In this study, we have examined the ECR films microscopically by EELS combined with TEM, in order to understand their physical origins of the macroscopic properties.

\section{Experimental Methods}

Thin carbon films were prepared by the ECR technique for three different bias voltages: $V_{\mathrm{B}}=0 \mathrm{~V},-75 \mathrm{~V}$, and $-120 \mathrm{~V}$. Details about the preparation technique were described previously. ${ }^{5)}$ The last two voltage settings were chosen from the region of interest: $-40 \mathrm{~V}<(-75 \mathrm{~V},-120 \mathrm{~V})<-140 \mathrm{~V}$. The films were deposited on a cleaved surface of sodium chloride at room temperature. Prior to each deposition, the substrate was irradiated by argon ions in order to clean its surface. The films were floated off from the substrate in distilled water, and taken onto a standard TEM microgrid covered with a holey amorphous carbon film (a-C). In addition to the ECR films, graphitized carbon and a-C were investigated for comparison.

EELS measurements and microscopic observations of the films were performed in a $200 \mathrm{kV}$ transmission-electronmicroscope (JEM 2010) attached with an EELS spectrometer (Gatan Digi-PEELS, model 766). Spectra were acquired from some circular areas of the film, each of which was $\phi 225 \mathrm{~nm}$ and determined by the selected-area-aperture. For the lowloss spectra, the energy resolution was $0.9 \mathrm{eV}$ and the momentum resolution was $0.72 \mathrm{~nm}^{-1}$. For the carbon K-edges, the energy resolution was $1.4 \mathrm{eV}$ and the momentum resolution was $1.44 \mathrm{~nm}^{-1}$ in order to improve the signal-to-noise ratio. No specimen was found to suffer from contamination. Most of the films were stable under electron irradiation. The signal intensity at the K-edge decreased during observations only for the film prepared at $V_{\mathrm{B}}=0$. When the exposure time 
was decreased for spectra acquisitions, this effect of irradiation damage became negligible.

The plasmon energy $E_{\mathrm{p}}$ was measured from the plasmon peak. The fraction of carbon atoms clustered with the $\mathrm{sp}^{2}$ bonds in the film was estimated from the carbon K-edge using the method proposed by Berger et al $^{4)}$ The relative ratio of the intensity under the characteristic $\pi^{*}$ peak to that under the entire K-edge was calculated in order to obtain the fraction. The energy window for the latter integration was $50 \mathrm{eV}$. The ratio was normalized for that of graphitized carbon, which was assumed to consist of $100 \% \mathrm{sp}^{2}$ bonding. The pre-edge background was removed, and each spectrum was deconvoluted by the corresponding low-loss spectrum. In low-loss spectra and the K-edges, the effect of multiple scattering and the instrumental broadening were removed by deconvolution when necessary. The orientation dependence of spectral features, if any, was averaged out by adding more than ten spectra which were acquired from different areas.

\section{Results}

\subsection{TEM images}

Figure 1 shows a low magnification TEM image of the ECR film which was prepared for $V_{\mathrm{B}}=-75 \mathrm{~V}$. The wavy pattern which spreads over $200 \mathrm{~nm}$ indicates that the film has textile structures. The minimum size of the structure was about $50 \mathrm{~nm}$, which was much larger than electronic mean free path of electrons in solid, so that the wavy structure is considered not to affect the electric properties of the film. The wavy pattern is probably related to the surface roughness of the substrate which was caused by irradiation of argon ions. The pattern observed in the ECR films for other bias voltages, i.e. $V_{\mathrm{B}}=0,-75$ and $-120 \mathrm{~V}$, were very similar to that in the figure.

At high magnification, the ECR films showed their difference in their finer atomic structures as seen in Fig. 2. When $V_{\mathrm{B}}=0 \mathrm{~V}$, the films appeared to be amorphous. For $V_{\mathrm{B}}=-75$

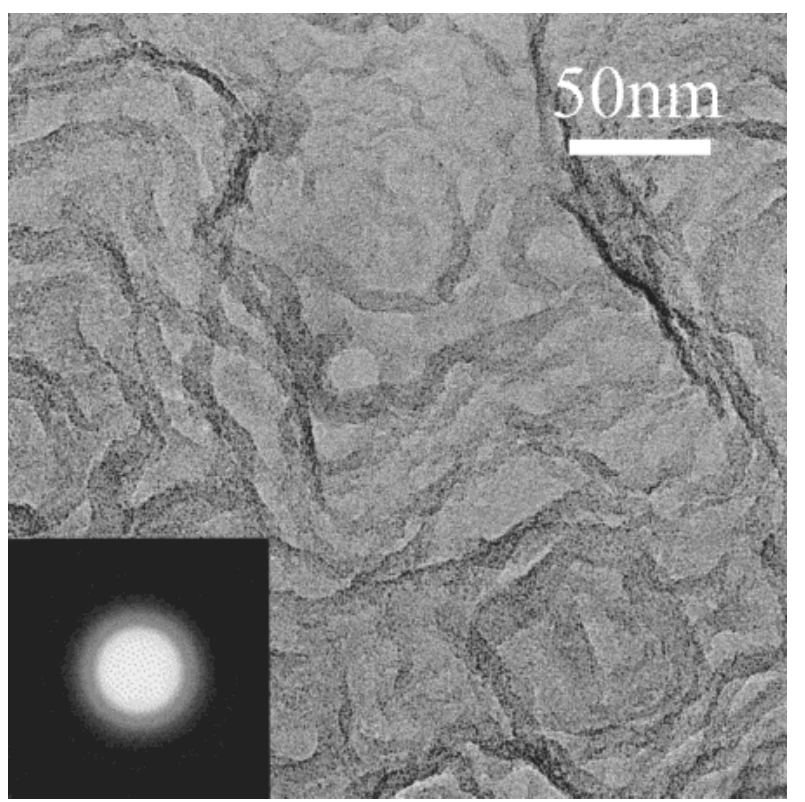

Fig. 1 TEM image of the ECR film prepared for the bias voltage of $-75 \mathrm{~V}$. A typical diffraction pattern recorded from the film is shown in the inset. and $-120 \mathrm{~V}$, the atomic structures were similar, i.e. most areas were amorphous-like but some were graphitized. Since the $(00,2)$ lattice fringes of graphite were sometimes observed in the ECR films for $V_{\mathrm{B}}=-75$ and $V_{\mathrm{B}}=-120 \mathrm{~V}$, atomic clusters such as aromatic rings (i.e. $\mathrm{sp}^{2}$ clusters), which can be a basic unit to build the graphite structure, could play important roles to form the ECR films.

Electron diffraction provided some information about ordered arrangement of carbon atoms. The diffraction pattern recorded from various parts of this film was diffusive concentric rings, i.e. the halo pattern, as shown in the inset. The diffraction patterns were the halo pattern for the other bias voltages, too. It was clear that the films were basically amorphous, but the film could possibly consist of fine crystallites of less than $1 \mathrm{~nm}$. In fact, for $V_{\mathrm{B}}=-75 \mathrm{~V}$, the diffraction pattern showed sharp spots, although it rarely happened. The spots appeared in positions corresponding to that of 00,2 reflection of graphitized carbon. This clarifies that graphite crystals existed in the film, which are small but still larger than the fine crystallites. Therefore, the ECR films may be rich in such a cluster as an aromatic ring. More detailed study can be done in order to distinguish amorphous structure and randomly distributed fine crystallites, using techniques such as high-resolution TEM or STEM or extended-energy-lossfine-structure spectroscopy. ${ }^{6}$

\subsection{Low-loss spectrum}

Figure 3 shows low-loss spectra that were recorded from the ECR films prepared in three different bias voltages. Spectra from graphitized carbon and amorphous carbon are also shown for comparison. As seen in the figure, there were two characteristic peaks in all spectra. The broad peak at around $20 \mathrm{eV}$ is the $(\pi+\sigma)$ plasmon i.e. the bulk plasmon, which corresponds to the collective excitation of all $(\pi+\sigma)$ electrons. The change in the peak position corresponds to that in the electronic density of the films, ${ }^{3)}$ and consequently that in the physical density when it is made of pure carbon. ${ }^{7)}$ The other one at around $4 \mathrm{eV}$ is the $\pi$ plasmon, which corresponds to the collective excitation of $\pi$ electrons. ${ }^{8)}$ This peak relates to $\pi \rightarrow \pi^{*}$ transitions, and its presence implies the existence of carbon clusters based on the $\mathrm{sp}^{2}$ bonding. ${ }^{9)}$ The energy positions for these two characteristic peaks are summarized in Table 1 for each specimen.

The surface plasmon can contribute to the low-loss spectrum since the ECR films were thin. The relative thickness $t / \lambda_{\mathrm{P}}$ of the films was estimated from the relative ratio of the zero-loss peak and the low-loss spectrum using the standard method. ${ }^{3)}$ Here $t$ is the thickness of the film and $\lambda_{\mathrm{P}}$ is the inelastic mean free path of plasmon excitation in carbon. For all the ECR films, $t / \lambda_{\mathrm{P}}$ was 0.1 and thus $t$ was $\sim 10 \mathrm{~nm}$ on the assumption that $\lambda_{\mathrm{P}}=100 \mathrm{~nm}$. The thickness of the film could be overestimated since the contribution from the surface loss was included in the spectrum. The presence of such surface losses, however, does not alter the following discussions and conclusions.

The plasmon peak the ECR films was observed at $18.6 \mathrm{eV}$, $17.6 \mathrm{eV}$ and $19.4 \mathrm{eV}$ for $V_{\mathrm{B}}=0 \mathrm{~V},-75 \mathrm{~V}$ and $-120 \mathrm{~V}$, respectively. These energies were lower by $\sim 10 \mathrm{eV}$ than those for carbon films which were $\mathrm{sp}^{3}$ rich. This is a sign that the fraction of $\mathrm{sp}^{3}$ bonding is small, since the plasmon energy is 


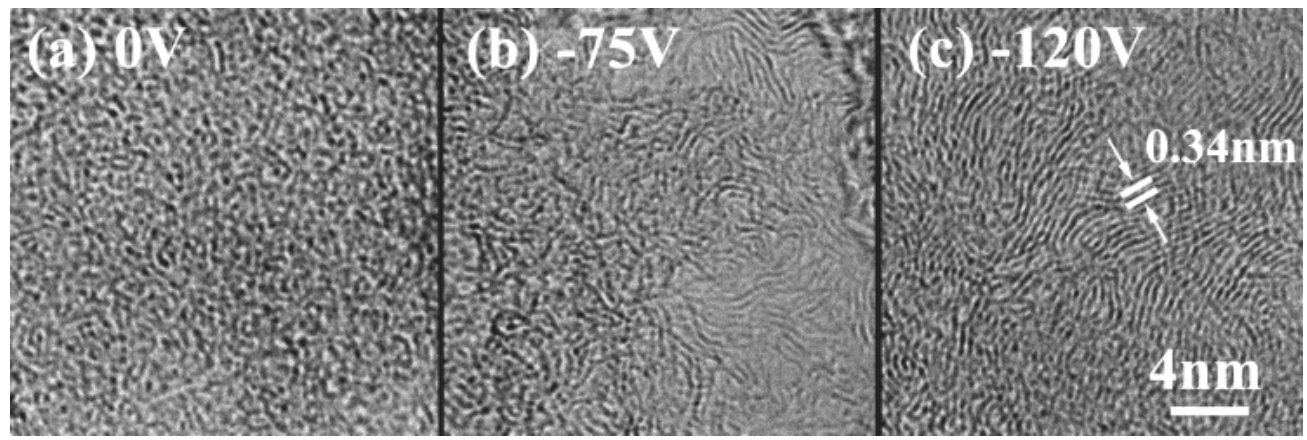

Fig. 2 High resolution TEM images recorded from ECR films for the three bias voltages; (a) $V_{\mathrm{B}}=0 \mathrm{~V}$, (b) $V_{\mathrm{B}}=-75 \mathrm{~V}$ and (c) $V_{\mathrm{B}}=-120 \mathrm{~V}$, respectively. The $(00,2)$ lattice fringe which corresponds to that of graphite is depicted in (c).

Table 1 The characteristics of the $\pi$ plasmon and the $\pi+\sigma$ plasmon in low-loss spectra for the ECR films.

\begin{tabular}{|c|c|c|c|c|c|}
\hline Samples & $\pi$ plasmon $(\mathrm{eV})$ & Area dependence & Intensity variation & $(\pi+\sigma)$ plasmon eV & Area dependence \\
\hline Graphitized C & 5.7 & No & Yes $( \pm 15 \%)$ & 22.2 & Yes (21-24) \\
\hline $\operatorname{ECR}\left(V_{\mathrm{B}}=-120 \mathrm{~V}\right)$ & 4.5 & Yes $(4.2-4.8)$ & Yes $( \pm 15 \%)$ & 19.4 & little \\
\hline $\operatorname{ECR}\left(V_{\mathrm{B}}=0 \mathrm{~V}\right)$ & 4.2 & Yes (3.2-4.4) & Yes $( \pm 15 \%)$ & 18.6 & little \\
\hline Amorphous C & 4.8 & No & No & 21.0 & No \\
\hline
\end{tabular}

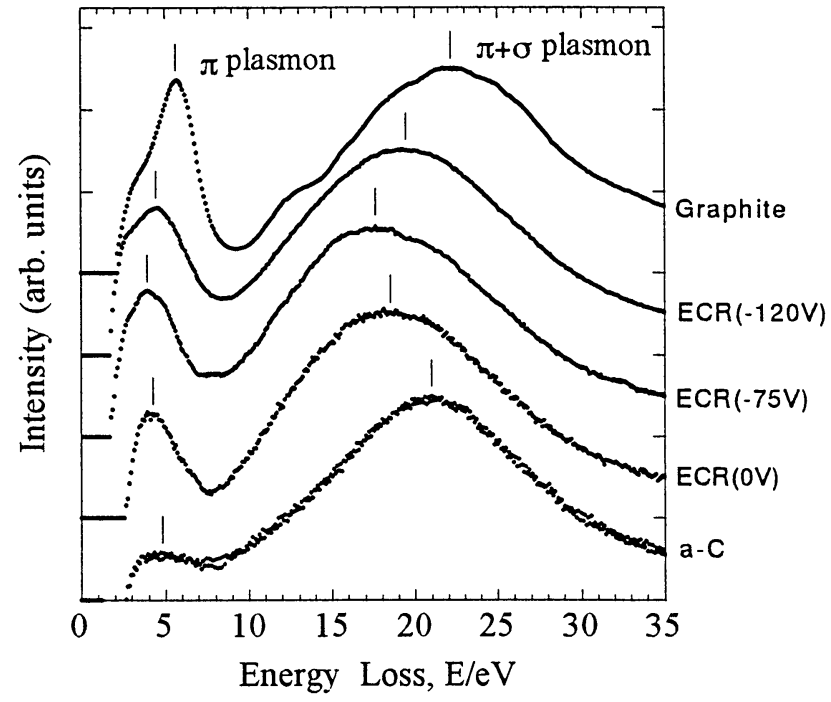

Fig. 3 Low-loss spectra recorded from ECR films. Spectra from graphitized carbon (top) and amorphous carbon (bottom) are also shown for comparison. The peak position of the $\pi$ plasmon and that of the $(\pi+\sigma)$ plasmon are depicted by short lines. The spectra are normalized at the $(\pi+\sigma)$ plasmon peak, and shifted vertically by a constant value for clarity. Data below $2.5 \mathrm{eV}$ are truncated because they are unreliable due to errors in zero-loss subtraction.

proportional to the fraction of $\mathrm{sp}^{3}$ bonding. ${ }^{10)}$ The energies for the ECR films were even lower by $\sim 2 \mathrm{eV}$ than that of graphitized carbon and that of amorphous carbon. These evidences suggest that the density of the ECR films is low and its fraction of $\mathrm{sp}^{3}$ bonding is small.

It is to be noted that the plasmon energy did not change monotonically as a function of the bias voltage for the ECR films. The plasmon energy decreased when $V_{\mathrm{B}}$ was changed from $-120 \mathrm{~V}$ to $-75 \mathrm{~V}$, but increased when $V_{\mathrm{B}}=0 \mathrm{~V}$. This suggests that the film for $V_{\mathrm{B}}=-75 \mathrm{~V}$ has the lowest density. The behavior of the plasmon peak position may relate to the changes in the spatial arrangement of the $\mathrm{sp}^{2}$ clusters in a short range; the clusters can take a large variety of well ordered arrangements.

Behaviors of the $\pi$ plasmon peak can provide alternative kind of evidences about the short-range ordering and atomic bonding. As seen in Fig. 3 and Table 1, the relative intensity of the peak for the ECR films was much higher than that for amorphous carbon, especially for $V_{\mathrm{B}}=-120 \mathrm{~V}$ and $-75 \mathrm{~V}$, and was close to that for graphitized carbon. This implies that there is a significant fraction of $\mathrm{sp}^{2}$ clusters.

Similar to the behavior of the $(\pi+\sigma)$ plasmon peak, the $\pi$ plasmon energy was the minimum when $V_{\mathrm{B}}=-75 \mathrm{~V}$. The energy of the $\pi$ plasmon was $4.5,4.0$ and 4.2 for $V_{B}=-120$, -75 and $0 \mathrm{~V}$, respectively. This may suggest that the density of $\pi$ electrons was the lowest for $V_{\mathrm{B}}=-75 \mathrm{~V}$, which is consistent with the analysis performed at the $\pi+\sigma$ plasmon peak.

\subsection{Carbon K-edge}

Figure 4 shows carbon K-edge spectra recorded from the ECR films. The edges from graphitized carbon and amorphous carbon are also shown for comparison. Spectra were normalized by the intensity at about $298 \mathrm{eV}$, and were aligned at the $\pi^{*}$ peak for energy calibration. All spectra have a peak at the onset of the K-edge, followed by a broad maximum that spreads between $290 \mathrm{eV}$ and $305 \mathrm{eV}$. The peak at $285.5 \mathrm{eV}$ is due to transitions from the core 1 s state to the $\pi^{*}$ states above the Fermi level. This peak implies the existence of $\mathrm{sp}^{2}$ bonded clusters in the area where spectra were recorded. The broad peak is due to transitions from $1 \mathrm{~s}$ to $\sigma^{*}$ states.

The fraction of the $\mathrm{sp}^{2}$ bonding was estimated from the $\pi^{*}$ peak using the quantitative technique of Berger et al. Data for graphitized carbon and that for amorphous carbon are also shown. All the ECR films had a $\mathrm{sp}^{2}$ fraction of more than $90 \%$ as shown in Table 2. Despite of differences in the $\pi^{*}$ 


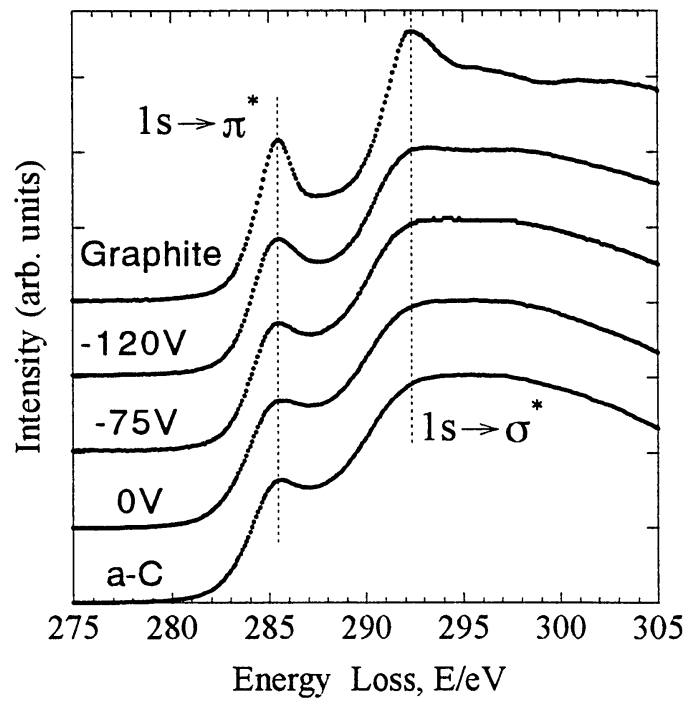

Fig. 4 Carbon K-edges recorded from the ECR films. Spectra from graphitized carbon and amorphous carbon are also shown for comparison. Each spectrum is normalized by the signal intensity at about $298 \mathrm{eV}$, and is aligned at the $\pi^{*}$ peak which is set to be $285.5 \mathrm{eV}$. Two vertical broken lines are drawn at the $\pi^{*}$ and $\sigma^{*}$ peaks in order to clarify their changes both in the energy position and the spectral features.

Table 2 The characteristics of the $1 \mathrm{~s} \rightarrow \pi^{*}$ peak in the K-edge for the ECR films.

\begin{tabular}{lcccc}
\hline Samples & $1 \mathrm{~s} \rightarrow \pi^{*}(\mathrm{eV})$ & $\begin{array}{c}\text { Area } \\
\text { dependence }\end{array}$ & $\begin{array}{c}\text { intensity } \\
\text { variation }\end{array}$ & $\mathrm{sp}^{2}(\%)$ \\
\hline Graphitized C & 285.5 & Yes $( \pm 10 \%)$ & Yes $( \pm 15 \%)$ & 100 \\
ECR $\left(V_{\mathrm{B}}=-120 \mathrm{~V}\right)$ & - & Little & Little & $>90$ \\
ECR $\left(V_{\mathrm{B}}=-75 \mathrm{~V}\right)$ & - & Little & Yes $( \pm 5 \%)$ & $>90$ \\
ECR $\left(V_{\mathrm{B}}=0 \mathrm{~V}\right)$ & - & Little & Little & $>90$ \\
Amorphous C & - & Little & No & $>90$ \\
\hline
\end{tabular}

peak, the $\mathrm{sp}^{2}$ fraction were quite similar in all the ECR films, which is consistent with the analysis at the $\pi$ plasmon peak.

It is known that the estimation by the Berger's method depends largely on the parameters such as the width of the energy window for data processing. ${ }^{11)}$ It was demonstrated that the absolute fraction of $\mathrm{sp}^{3}$ bonding could be more accurately estimated. For the $\mathrm{sp}^{2}$ fraction, however, only a relative value appears to be meaningful. In the present study, it has been clarified that the fraction of $\mathrm{sp}^{2}$ bonding is significant in the ECR film. The accuracy of the results is still limited by that of the reference spectrum from graphitized carbon and by that of the separation of the contribution from the $\pi$ states and that from the $\sigma$ states.

In general, the K-edge of the ECR films looked quite similar to that of amorphous carbon. However, there are some points to be noticed. When the bias voltage was lower, the $\pi^{*}$ peak was sharper and its relative intensity was closer to that of graphitized carbon. In addition, a small bump started being visible at about $292 \mathrm{eV}$. Since the fraction of the $\mathrm{sp}^{2}$ clusters is significantly large in the film, these evidences could suggest that a narrower energy band was formed; more fraction of the $\mathrm{sp}^{2}$ clusters are ordered at the lower voltage i.e. $V_{\mathrm{B}}=-75$ and $-120 \mathrm{~V}$. Such an interpretation can be consistent with the low-loss spectroscopy.

It was suggested in Section 3.1 that fine crystallites of graphite could be included in the ECR film for $V_{\mathrm{B}}=-75$. Such a difference in the atomic structures seemed to be reflected in the behavior of the $\pi^{*}$ peak. Unlike the other Kedges, the relative intensity of the peak varied by $\pm 5 \%$ for $V_{\mathrm{B}}=-75 \mathrm{~V}$. This variation was less than that of graphitized carbon but more than that of other ECR films. This variation must be due to the variation of the contribution from the fine graphite crystallites which have a strong orientation dependence in the $\pi^{*}$ peak.

The ECR film prepared for $V_{\mathrm{B}}=0 \mathrm{~V}$ only suffered from noticeable irradiation damage as mentioned in Section 2. At the C K-edge for $V_{\mathrm{B}}=0 \mathrm{~V}$, the $\pi^{*}$ peak was broader than that for amorphous carbon. The small bump was not visible at about $290 \mathrm{eV}$. The gap immediately after the $\pi^{*}$ peak was more filled than the other K-edges. At the same time, the fraction of $\mathrm{sp}^{2}$ bonded clusters is considered to be large, as shown in the low-loss spectroscopy. The above differences observed at the K-edge, therefore, could be due to the lack of cluster ordering already mentioned above, which may result in the mixing of the $\pi$ and the $\sigma$ states. This may consequently result in weaker carbon bonds, and in the damage.

\section{Discussions}

The behaviors of the $(\pi+\sigma)$ plasmon and $\pi$ plasmon were not as systematic as the $\pi^{*}$ peak at the C K-edge. This nonmonotonic behavior of the $(\pi+\sigma)$ and $\pi$ plasmon energies may relate to the ordering of $\mathrm{sp}^{2}$ clusters while they are deposited on the substrate. For a lower bias voltage, the bombardment of ions onto the substrate should be more energetic. When $V_{\mathrm{B}}=-100 \mathrm{~V}$, the deposition rate of the film decreased by $50 \%$ because of the increase in the etching efficiency. On the other hand, the arrangement in $\mathrm{sp}^{2}$ configuration has a lower energy than that in $\mathrm{sp}^{3}$ configuration. ${ }^{12)}$ Therefore, the non-monotonic behavior of the plasmon peak could reflect the process that atoms are self-ordered to form a structure with a rather low physical density by minimizing the total energy.

The ECR films may have more than one phase, each of which is different, for example, in the ordering of clusters in a medium-range distance. If this is the case, the electron density could be different depending on the area. It was found that the $(\pi+\sigma)$ plasmon energy varied little for any ECR films when the low-loss spectrum was recorded from different areas; the result is summarized in Table 1. This means that the averaged electron density of the films does not vary much when the spectrum recorded from the circular area of $\phi 225 \mathrm{~nm}$. It also suggests that phase-separation which accompanies a large variation in the electronic density is unlikely, and, if any, the spatial size of each phase would be less than $\phi 225 \mathrm{~nm}$. For $V_{\mathrm{B}}=-75 \mathrm{~V}$, small graphite crystals do not appear to contribute significantly to the spectrum.

In Section 3.2, the behavior of the $\pi$ plasmon peak was interpreted from the difference in the electronic density. However, this density was the averaged value over some areas. In fact, the energy position and the shape of the $\pi$ peak varied depending on where spectra were acquired. Its relative intensity varied by $\pm 15 \%$. The peak shifted over $\sim 1 \mathrm{eV}$. The peak shape varied, too. These changes did not appear systematically.

In order to understand such complicated behavior of the $\pi$ 
plasmon peak, we may need to take into account more localized information about electronic structures and atomic bonding. While the $\pi$ plasmon peak has been interpreted as a collective excitation in the present study, there is a possibility that the peak is due to single-electron excitation instead. This ambiguity can be clarified by measuring the dispersion of the peak. ${ }^{9)}$ Other points are, for example, the differences in the degree of $\mathrm{sp}^{2}$ clustering, their orientations with respect to the beam, and the differences in the electronic transitions due to the variation of a local band structure.

Unlike carbon films prepared by other techniques such as the RF sputtering and the filtered-cathode vacuum arc, which are $\mathrm{sp}^{3}$ rich, the film prepared by the ECR techniques on a sodium chloride substrate was rich in $\mathrm{sp}^{2}$ clusters. The ECR film prepared on silicon substrate is also rich in $\mathrm{sp}^{2}$ clusters. The atomic structures of the film based on the $\mathrm{sp}^{2}$ clusters seem to be finely controlled by a simple experimental parameter such as a bias voltage $V_{\mathrm{B}}$. Since $\pi$ electrons of the cluster relate to the electric properties and $\sigma$ electrons do to the mechanical properties, this control ability may allow fine tuning of electric and mechanical properties of the film as potentially demonstrated for the film on silicon substrate.

\section{Conclusions}

Carbon films were prepared by the ECR technique for three different bias voltages using a sodium chloride substrate, and characterized by EELS in a conventional transmission electron microscope. The physical density of the films was found to be lower than that of graphitized carbon and that of amorphous carbon. The fraction of the clusters was more than $90 \%$ for all of the ECR films, while the films were basically amorphous. The difference in the bias voltage seemed to result in ordering of the $\mathrm{sp}^{2}$ clusters; i.e. they could be more ordered for $V_{\mathrm{B}}=-75 \mathrm{~V}$ and $-120 \mathrm{~V}$ than for $V_{\mathrm{B}}=0 \mathrm{~V}$. Using the ECR technique, the properties of the protective films may be finely tailored by a simple experimental parameters such as a bias voltage.

\section{Acknowledgments}

The present authors would like to thank Prof. M. Morinaga of Nagoya University for his kind encouragement and discussion about the study of local electronic structures of light elements. The present study is partly supported by Grant-in-Aid for Research on Priority Area(B), "Localized Quantum Structure (Area 751)", Grant-in-aid for Exploratory Research (\#13875005) and Grant-in-Aid for Scientific Research on Priority Area (A), "Global Interface Integration" (\#13025224) of the Ministry of Education, Science, Sports and Culture.

\section{REFERENCES}

1) S. Umemura, Y. Andoh, S. Hirono, T. Miyamoto and R. Kaneko: Philos. Mag. A 74 (1996) 1143-1157.

2) S. Hirono, S. Umemura, M. Tomita and R. Kaneko: Appl. Phys. Lett. 80 (2002) 425-427.

3) R. F. Egerton: Electron energy-loss spectroscopy in the electron microscope 2nd ed., (Plenum Press, New York, 1996) pp. 154-165.

4) S. D. Berger, D. R. McKenzie and P. J. Martin: Philos. Mag. Lett. 57 (1988) 285-290.

5) S. Hirono, S. Umemura, Y. Andoh, T. Hayashi and R. Kaneko: IEEE Trans. Magn. 34 (1998) 1729-1731.

6) J. Fink, T. Mueller-Heinzerling, J. Pflueger, A. Bubenzer, P. Koidl and G. Crecelius: Solid State Comm. 47 (1983) 687-691.

7) L. B. Leder and J. A. Suddeth: J. Appl. Phys. 31 (1960) 1422-1426.

8) Y. H. Ichikawa: Phys. Rev. 109 (1958) 653-657.

9) J. Fink: Adv. Electr. Electron Phys. 75 (1989) 121-232.

10) P. J. Fallon, V. S. Veerasamy, C. A. Davis, J. Robertson, G. A. J. Amaratunga, W. I. Milne and J. Koskinen: Phys. Rev. B 48 (1993) 4777-4782.

11) J. Bruley, D. B. Williams, J. J. Cuomo and D. P. Pappas: J. Microsc. 180 (1995) 22-32.

12) M. T. Yin and M. L. Cohen: Phys. Rev. B 29 (1984) 6996-6998. 